\title{
METODOLOGÍA DE LA ENSEÑANZA BASADA EN COMPETENCIAS
}

\author{
Jesús Martín Cepeda Dovala \\ Universidad Autónoma del Noreste, México
}

\section{INTRODUCCIÓN}

Nuestra propuesta es el resultado de 13 años de experiencia aplicando el modelo de educación basada en competencias en el nivel de educación superior y de posgrado en la Universidad Autónoma del Noreste y de la Universidad Autónoma de Fresnillo.

Nuestro objetivo es delimitar las fases del proceso de la metodología de la enseñanza basada en competencias de programas en el contexto del diseño curricular, por lo que partimos, entre otros supuestos básicos, de que el proceso de evaluación, el de programación y planeación se interrelacionan y se deben producir paralelamente; es decir, la evaluación puede realizarse en cualquier etapa o fase de la programación, en un proceso de retroalimentación permanente.

Por otra parte, damos por sentado que el programa se basa en la identificación y determinación de necesidades de la sociedad, a partir de una evaluación del contexto social y educativo del centro escolar donde se va a desarrollar. A la vez que consideramos como finalidad general de la metodología de la enseñanza de programas basados en competencias la toma de decisiones para la mejora del proceso de intervención y del logro de resultados.

En definitiva, la metodología de la enseñanza de un programa basado en competencias y de alta dirección consiste en realizar un seguimiento a lo largo de todo el proceso, que permita obtener información acerca de cómo se está llevando a cabo, con la finalidad de reajustar la intervención orientadora, de acuerdo con los datos obtenidos. Es necesario tener en cuenta en toda evaluación que ésta debe ajustarse a las características del contexto donde el programa se esta aplicando.

La evaluación de un programa basado en competencia de alta dirección tiene una metodología concreta, que permite evidenciar si la intervención o proceso de actuación llevado a cabo es no sólo correcto sino eficaz.

Así, la evaluación de un programa no es un mero acto puntual, sino que sirve para una mejora continuada del programa, la hemos de entender como un proceso. Este proceso de evaluación se encuentra íntimamente relacionado con la programación y planeación del programa, pudiendo de este modo efectuar una constante retroalimentación. En este sentido, en nuestra propuesta, el proceso de la metodología de la enseñanza se puede fijar en seis momentos o tipos de evaluación:

- Evaluación de necesidades.

- Especificación de competencias.

- Determinación de componentes y niveles de realización.

- Identificación de procedimientos para el desarrollo de competencias.

- Definición de evaluación de competencias. 
- Validación de competencias.

En definitiva, la finalidad general de la evaluación es tomar decisiones de cambio y mejora a lo largo del proceso y tras finalizar la intervención del programa.

Aunque nuestra propuesta va destinada inicialmente al profesional que en su práctica pretende evaluar un programa que diseña o adapta para cubrir las necesidades de un contexto determinado, en la línea de lo que hemos afirmado anteriormente de entender la evaluación como núcleo de todo el proceso de planeación y programación, pensamos que es perfectamente aplicable en sus orientaciones generales a otras situaciones posibles en la práctica educativa. En todos los casos el diseño de metodología y evaluación se deberá adaptar en sus cuestiones evaluativos, contenido y tipo de evaluación, a los datos de que se disponga. En la exposición de cada una de las fases, iremos especificando las posibles adaptaciones a estas situaciones.

\section{FASES EN EL PROCESO DE LA METODOLOGÍA

\section{PROCESODE LA EDUCACÓN BASADAEN COMPETENAAS}

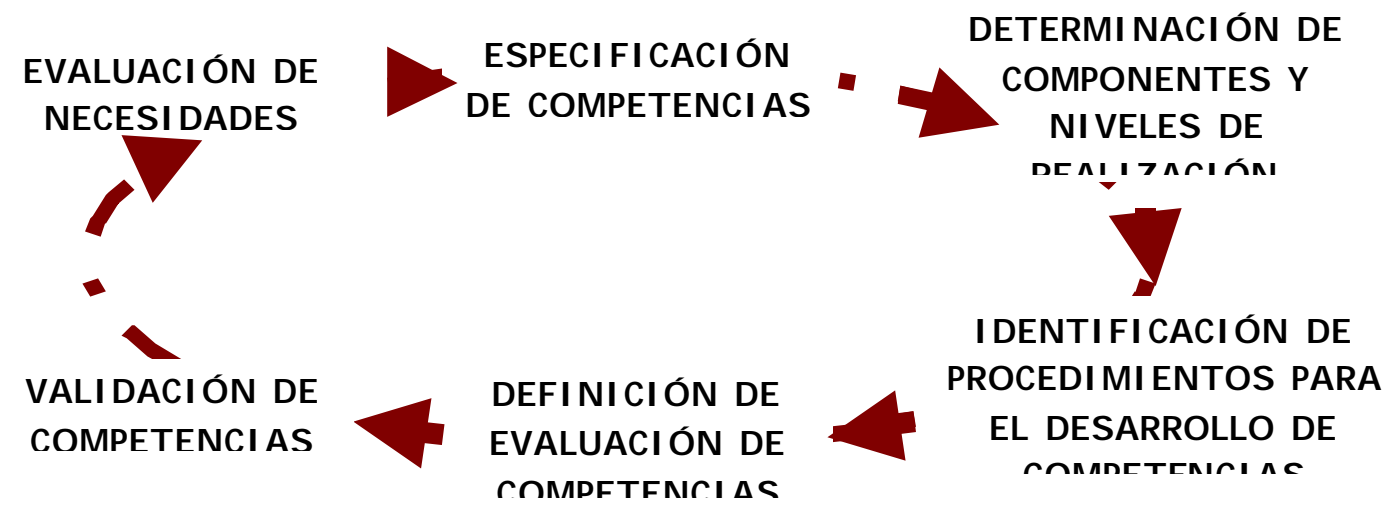

ES TE PRO CES O DEBE LLEVARSE A CABO EN TODAS LAS CLAS ES, PARA DET ERMINAR LO

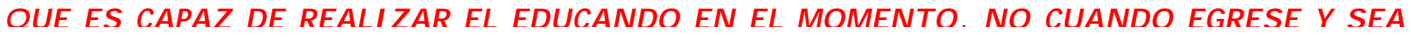

\section{1a Fase: Evaluación de necesidades}

Antes de iniciar el diseño de la evaluación de un programa basado en competencias de alta dirección y, por tanto, en el momento de iniciar la propia planeación y diseño del mismo hay que fijar los supuestos teóricos-previos en los que se va a basar dicha evaluación. En ellos se debe especificar, entre otros aspectos:

- Exponer propósitos e importancias de la evaluación.

- Papel del evaluador: tipo de valuación: interna, externa, mixta.

- Destinatarios, receptores de la evaluación. 
- Tipo de información que se va a dar.

- Diseño de investigación en la evaluación.

- Utilidad, adecuación de la evaluación en el contexto a que va dirigido, lo que en cierta medida matizará los destinatarios y los responsables de tomar decisiones sobre el programa, teniendo en cuenta tanto la precedencia de la demanda del programa como la de la evaluación.

En aspectos, junto con las características del programa y las del contexto en que se va a llevar a cabo el mismo, nos servirán para determinar el modelo y tipo de evaluación en el diseño de ésta, una vez que se valide el programa y podamos pasar a precisar la metodología de evaluación.

- Justificación.

- Competencias necesarias.

- Qué tipo de competencias es prioritarios a desarrollar en el alumno, en esta materia.

- Cómo aplicar dichas competencias en una situación real de trabajo.

- Utilidad que representan dichas competencias en el desarrollo profesional y social.

- Importancia de obtener ciertas habilidades o competencias adicionales.

Para la evaluación de necesidades es importante:

Investigar cuáles son las competencias necesarias para que el educando pueda desempeñar un rol profesional, para ello se requiere:

- Estudio del entorno:

a) Marco: Social, político y económico

b) Micro: Dentro del salón de clase

- Determinar qué campos o áreas del conocimiento son importantes.

- Definir las características del egresado a través de un estudio del rol que va a desempeñar.

Los dos esquemas anteriores nos llevan a la conclusión, que en el planteamiento de un programa necesitamos de una constante actualización dado que el entorno social, político y económico, es un entorno que sufre constantes cambios, y aunque nuestros programas anticipan exigencias futuras, deberán estarse adecuando inicialmente dentro de la revisión de cada quinquenio, como la ha venido haciendo, y estudiar luego si no convendrá un período de previsión, en un período más corto (tres años por ejemplo), desde el momento que ese entorno ilumina y determina nuestra realidad micro-social del salón de clases, de nuestra institución, de los campos del conocimiento, de los roles a jugar y consiguientemente de las características del egresado de la Universidad.

Por otro lado, la evaluación de necesidades exige cada uno de esos componentes: justificación, competencias, tipo de competencias, aplicación, utilidad, Importancias, todo eso expresado en forma muy sintética según ya se observa en la mayoría de nuestros programas.

\section{2aㅗ Fase: Especificación de competencias}


Esta segunda fase, junto con la siguiente, corresponde al momento de evaluación del diseño del programa. Aunque algunos autores prefieren considerar a ambas como una fase única de evaluabilidad de la evaluación, nuestra experiencia en la aplicación del modelo nos reafirma en la decisión de considerarlas como dos fases con entidad propia aunque interrelacionadas dentro del momento de la evaluación del diseño, puesto que, al margen de aspectos metodológicos, cada una de ellas responde a una cuestión básica y secuenciada.

Analizar si el enfoque u orientación que se dio a la carrera en cuestión, enfoque plasmado en el plan de estudios respectivo, sigue siendo válido o necesita modificarse.

Para este análisis se necesita tener en cuenta por una parte, la relación entre los rasgos del profesionista (que se pretende formar con un plan de estudios dado) y las necesidades sociales dadas y, por otra, los cambios que haya habido en conocimientos científicos y en las tecnologías que el egresado se supone dominará y utilizará en el ejercicio de su profesión.

Los enunciados de competencias deben ser:

- Específicos

- Reales

- Que precisen una habilidad

- Redactados en tiempo presente

- Jerarquizados por áreas de conocimiento:

- Definir qué es lo que el educando necesita de manejar de esa rama del conocimiento.

- Definir cuánto se la va a dar de cada área dependiendo de las características de los educandos, la carrera y necesidades de egreso.

\section{3를 Fase: Determinación de componentes}

En esta tercera fase de validación de la evaluación específicamente se pretende responder a la cuestión básica de si el programa reúne las condiciones para poder ser evaluado. En esta fase se pretende comprobar que los elementos formales están diseñados de tal forma que pueden ser evaluados, que reúnen los requisitos mínimos para que puedan pasar aceptablemente, los criterios, valoraciones, diseños y análisis propios de la evaluación de programas.

COMPETENCIAS PRINCIPALES PARA EL DESEMPEÑO PROFESIONAL

\begin{tabular}{|c|l|}
\hline CONCEPTUALES & $\begin{array}{l}\text { Dominio del conjunto de conocimientos teóricos necesarios que } \\
\text { sustentan un rol o una carrera. }\end{array}$ \\
\hline METODOLOGíCAS & $\begin{array}{l}\text { Maneras, procedimientos, métodos y técnicas especializadas que } \\
\text { requiere el desempeño profesional. }\end{array}$ \\
\hline HUMANAS & $\begin{array}{l}\text { Desarrollo de habilidades humanas, de comunicación e interacción, } \\
\text { requeridas para el desempeño profesional. }\end{array}$ \\
\hline ALTA DIRECCIÓN & $\begin{array}{l}\text { Capacidad para la auto-dirección, responsabilidad, solución de problemas } \\
\text { y toma de decisiones. }\end{array}$ \\
\hline
\end{tabular}


El Dr. Lloyd McCleary nos ofrece una definición de competencia y dice: "la competencia es definida como la presencia de características o la ausencia de incapacidades que hacen a una persona adecuada o calificada para realizar una tarea específica o para asumir un rol definido".

De lo anterior se deduce que una persona es competente cuando:

- Conoce cuales son sus capacidades tiene.

- Puede demostrar lo que sabe.

- Sobresale del resto por su capacidad para desarrollar procesos terminales.

Como podemos observar, una persona competente tiene características que lo conducen al autoanálisis de sus posibilidades de desarrollo así como de sus carencias, pero para llegar a este punto de formación, el docente debe utilizar el modelo de enseñanza que le permita tanto a él como a su alumno conocer y demostrar los logros alcanzados con relación a competencias.

Decirle a un alumno que él será competente para conocer o realizar un proceso es lo más recomendable; basta con que utilicemos un verbo inicial y le daremos al joven la pauta para alcanzar la competencia.

Antes de mencionar los verbos que se pueden utilizar para definir las competencias a lograr, es necesario considerar cómo el modelo educativo divide las competencias.

Para el ser humano, que es una entidad compleja y de comportamientos variados, se han contemplado tres tipos de competencias:

\section{Conceptuales}

A este tipo de competencias, se les define como el dominio que el estudiante debe tener sobre el conjunto de conocimientos teóricos necesarios, que sustentan una materia. En este grupo encontramos muchos de los conocimientos que deben ser conocidos por el alumno; hablamos de conceptos, teorías, tratados, que servirán como sustento a otros conocimientos o procesos más complejos.

\section{Metodologías}

Las competencias metodológicas, son aquéllas que indican al estudiante los elementos que habrá que disponer para obtener el conocimiento, procesos, pasos a seguir, métodos, técnicas o formas de hacer algo. Para este tipo de competencias el alumno conocerá, comprenderá o aplicará un proceso claro, es decir, que le llevarán a un resultado sí lo sigue de manera correcta.

\section{Humanas}

Dentro de las áreas del conocimiento, el alumno verá la necesidad desarrollar competencias que le permitirán desempeñarse en su campo laboral, donde utilizará los conocimientos de índole general y formativa que se encuentran en el plan de estudios como parte de su formación integral.

\section{4aㅡ Fase: Identificación de procedimientos para el desarrollo de competencias}

El temario de la materia, organizado por temas y subtemas según la importancia y jerarquía de cada rubro. 
El término contenido es utilizado para referirnos a todo aquello que puede ser objeto de aprendizaje: conocimiento, habilidad, proceso, etc.

Cuando el docente conozca el programa e inicie la dosificación de su curso, obtendrá los siguientes beneficios:

En el momento mismo de organizar los temas, el maestro tiene la oportunidad de observar que tópicos son más importantes para poner en ellos mayor énfasis.

Una vez establecidos en la dosificación y organizados lógicamente el maestro puede planear una estrategia de instrucción, es decir, puede diseñar un procedimiento adecuado para que el alumno logre la competencia y el nivel deseado.

El maestro obtiene una visión general del curso, lo que permite que tanto el alumno él mismo, planeen mejor su actividad y utilicen eficientemente los recursos de que disponen.

Cuando el alumno tiene una idea panorámica de todo el curso, puede ir relacionando unos temas con otros ya visto o que sabe que pronto estudiará. Es motivante para el que aprende y le ayuda a retener por más tiempo lo aprendido.

Metodología para el logro de competencias

Además de que catedrático cuenta con un repertorio didáctico propio para determinados contenidos, se sugieren la metodología de que elabore la dosificación en su asignatura ya que este se convierte en una herramienta útil para el logro de competencias y niveles de aprendizaje dentro del salón de clase.

En esta fase de evaluación de proceso el análisis de los datos y poder tomar decisiones. El análisis girará en torno a la marcha del programa tanto en su adecuación a la programación previa como a los aspectos dinámicos y de relación del mismo. Tiene, por tanto, un carácter formativo e implica la realización de evaluaciones después de cada clase que permitan la retroalimentación del programa. En cada una de ellas se puede distinguir como fases sucesivas, la evaluación de la implementación y la evaluación del desarrollo.

\section{- Evaluación de la implementación}

Deberá compararse, por una parte, el plan de estudios teórico con los resultados del análisis de la situación de cada carrera con respecto a los cuatro factores. Además, deberá compararse, el plan de estudios real, tal como se está instrumentando con el mismo análisis de los cuatro factores. Y finalmente, deberán compararse entre sí.

Consiste en evaluar <qué> está funcionado del programa una vez que se ha puesto en marcha, es decir, la instrumentalización del programa de intervención, su puesta en práctica siguiendo las etapas y esquemas teóricos previamente concretos. El fin último de esta fase de evaluación, es contrastar si hay o no discrepancias entre el diseño y la realidad: y en caso afirmativo, realizar la adaptación pertinente, redefinir el programa para lograr su óptima y adecuada puesta en marcha.

En esta fase nos centramos en cinco aspectos que no buscan otra cosa que la adecuación e identificación de las actividades diseñadas. A partir de estos aspectos, especificaremos los criterios e indicadores de evaluación. 
Las dimensiones a las que nos referimos son:

- Cobertura del programa.

- Realización de actividades: Se han llevado a la práctica todas y cada una de las actividades planeadas.

- Ejecución de la temporalización: Muy relacionada con la anterior, hace referencia que se han llevado a cabo las actividades previstas en los períodos de tiempo prefijado, es decir hay un ajuste entre la ejecución real y la planeación diseñada.

- Funciones de los agentes implicados: Cada agente implicado en el programa ha llevado a la práctica las actividades de las que era responsable, tal y como se diseñó.

- Utilización de los recursos disponibles: Se han empleado los recursos materiales y humanos que se habían previsto.

\section{5a Fase: Definición y evaluación de competencias}

Igual que la metodología, este elemento estructural de los programas está dispuesto para el docente a nivel de políticas y algunas sugerencias.

Los elementos de un programa de contenidos tomados en cuenta hasta este momento, son esenciales para la dosificación.

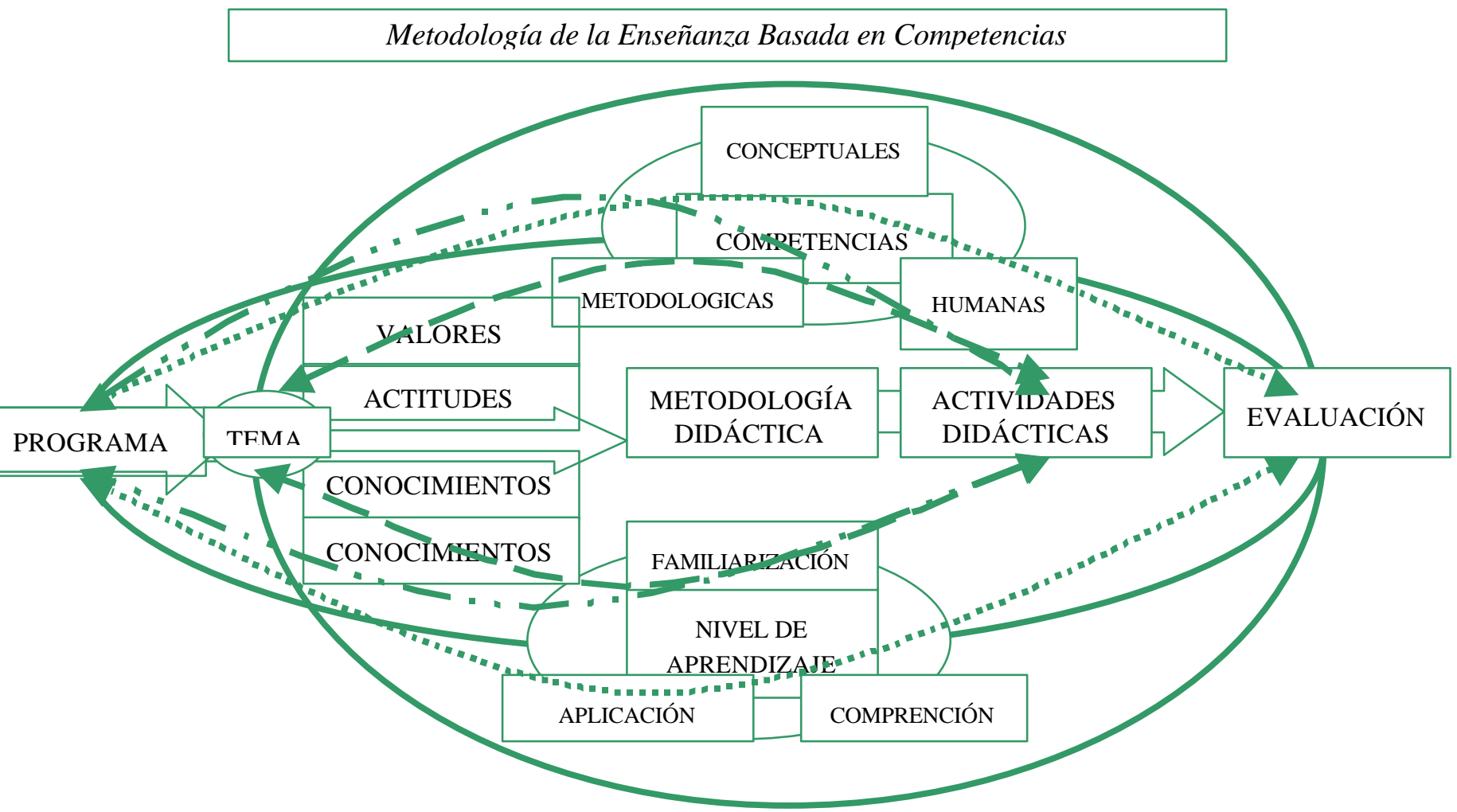




\section{Fase: Validación de competencias}

La dosificación de contenidos hasta este momento resulta de mucha utilidad para el trabajo del catedrático, puede en el acto trabajar en la enseñanza, guiándose con las competencias enunciadas, sin embargo, falta considerar un elemento muy importante, como la especificación el nivel de aprendizaje al que el alumno deberá llegar a través de la metodología y las actividades que el docente previamente señaló para el logro de las competencias.

El nivel de aprendizaje o categoría cognoscitiva, como suele llamarle Benjamín Bloom en su obra sobre taxonomía de los objetivos, consta de tres niveles en el Modelo de Educación Basada en Competencias característica de la Universidad del Autónoma del Noreste.

El nivel de aprendizaje establecido por el catedrático está determinado por el contenido temático y sus características, la profundidad y la extensión con que este será tratado de acuerdo a las competencias y niveles establecidos previamente en el programa de contenido de la materia.

Es importante definir cada uno de los tres niveles para la mejor comprensión de los mismos:

\section{Familiaridad}

Forma más elemental de conocer algo y poder conversar sobre ello.

El nivel de familiaridad implica que el alumno maneje información y datos concretos acerca de un tema específico con una profundidad que puede ser memorística y de extensión que le permita tener continuidad a una serie de ideas claras y precisas.

\section{Comprensión}

Permite al estudiante modificar la información original que ha recibido y transformarla en forma paralela para luego hacerla más significativa y almacenarla o transmitirla a otra persona.

\section{Aplicación}

Requiere de los dos niveles anteriores e implica el uso de información conocida y aprendida en situaciones nuevas, utilizando los recursos y herramientas que dicha información proporciona; esto se da a través de la transferencia de conocimiento adquirido.

\section{REFERENCIAS BIBLIOGRÁFICAS}

ARGUELLES. Antonio, Complilador en Competencia laboral y Educación Basada en Normas de Competencia. Editorial Limusa, Noriega. Primera edición, 1996. México.

BARRON Tirado. Concepción, ROJAS Moreno. lliana, Formación en Competencias Profesionales. Pensamiento Universitario, tercera época 91. Primera edición 2000 Universidad Autónoma Nacional de México. Centro de Estudios sobre la Universidad.

CePEDA Dovala. Jesús Martín, (2004) Metodología de la Enseñanza Basada en Competencias Libro por publicarse en Editorial Tópicos Culturales Á . A.R.C.D. Editor, Saltillo, Coahuila. M éxico. (Derechos de autor e ISBN en trámite).

CEPEDA Dovala. Jesús Martín, Modelo curricular basado en competencias de la Universidad Autónoma del Noreste, Saltillo, Coahuila, México, 2001. Universidad Autónoma del Noreste. 
DuCCl, Angélica, El enfoque de competencia laboral en la perspectiva internacional, en CONOCER, Formación basada en competencia laboral: situación actual y perspectivas. México, POLFORM/OIT/CINTERFOR/CONOCER, 1997.

GAGO Huguet. Antonio, Elaboración de Cartas Descriptivas: Guía para preparar el programa de un curso, Ed. Trillas, 1987.

GONCZl. Andrew, y ATHANASOU. James, Instrumentación de la educación basada en competencias. Perspectivas de la teoría y práctica australiana.

KOBINGER. Nicole, El sistema de formación profesional y técnica por competencias desarrollado en QUEBEC.

MATHENY Dillman y RaHMLOW, Cómo Redactar Objetivos de Instrucción. Ed. Trillas, 1983.

MERTENS, Leonard, Sistemas de competencia laboral: surgimiento y modelos, en CONOCER, Formación basada en competencia laboral: situación actual y perspectivas. México, POLFORM/OIT/CINTERFOR/CONOCER, 1997.

MORFIN. Antonio, La nueva modalidad educativa: Educación Basada en Normas de Competencia.

NAVA Jaimes. Arturo, Profesor investigador. Coordinador General de Universidades Tecnológicas de la Subsecretaria de Educación Superior e Investigación de la Secretaría de Educación e Investigación Científica. (SEISIC-SEP).

OVANDO Antelo. Martha, Educación basada en la competencia. CENAPRO, 1981. México. D.F.

AGUILAR, Må .J. y ANDER-EGG, E. (1992). Evaluación de servicios y programas sociales. Madrid: Siglo XXI.

ALVIRA, F. (1991). Metodología de evaluación de programas. Madrid: CIS-Cuadernos Metodológicos.

BeRK, R.A. y RosSI, P.H. (1990). Thinking About Program Evaluation. Sage : Newbury Park.

HeRnánDEZ, J. Y Martínez, P. (1994). Pautas para la evaluación de un programa de orientación. Revista de Investigación Educativa, (23), 598-604

HERNÁNDEZ, J.Ma ! y Rubio, V.J. (1992). Análisis de la evaluabilidad: paso previo de la evaluación de programas. Bordón, 43 (4). 297-405.

HERR, E.L. (1976). Counseling: Accountability, reality, credibility. Journal of Counseling Services, 1, 14-23.

Municipio, P. (1992) La evaluacióOn segmentada de programas. Bordón, ,43 (4),

375-395.

UNIVERSIDAD AUtÓNOMA DEL NORESTE (UANE). Documentos internos. Revisión Curricular y Estructura Académico-Administrativa. Plan AIDA 1996. 


\title{
Contactar
}

Revista lberoamericana de Educación

\author{
Principal OEI
}

